\title{
La réception de l'œuvre de Claude Simon en Espagne : une contribution provisoire
}

\section{Manuel A. Tost Planet}

\section{(2) OpenEdition}

1 Journals

\section{Édition électronique}

URL : https://journals.openedition.org/ccs/645

DOI : $10.4000 /$ ccs. 645

ISSN : 2558-782X

Éditeur:

Presses universitaires de Rennes, Association des lecteurs de Claude Simon

\section{Édition imprimée}

Date de publication : 31 décembre 2009

Pagination : 121-141

ISBN : 9782354120580

ISSN : 1774-9425

Référence électronique

Manuel A. Tost Planet, «La réception de l'œuvre de Claude Simon en Espagne : une contribution provisoire », Cahiers Claude Simon [En ligne], 5 | 2009, mis en ligne le 21 septembre 2017, consulté le 17 mai 2021. URL : http://journals.openedition.org/ccs/645 ; DOI : https://doi.org/10.4000/ccs.645 


\title{
La réception de l'œuvre de Claude Simon en Espagne : une contribution provisoire
}

\author{
Manuel A. TOST PLANET
}

En janvier 2005, j'ai eu l'occasion, dans le cadre du séminaire Claude Simon, de tenter une première approche de «la réception de Claude Simon en Espagne ». C'est en partie la transcription de cette intervention (dans une version modifiée et augmentée) qui est présentée ici. On y conserve le sous-titre donné alors à l'exposé (« Une contribution provisoire ») car on est convaincu que, pour ce genre d'étude, il est prudent de laisser le temps faire son lent travail de fixation et de décantage : la perception que l'on a, en un lieu et un temps, d'un auteur ou d'une ouvre (ou de certains aspects d'une œuvre, comme c'est le cas ici), peut connaître des avatars nombreux.

On s'en tiendra, pour ce qui concerne cette réception, à ce qui est plus ou moins traditionnel dans le genre : 1) les questions touchant à la traduction des œuvres et au phénomène éditorial (la fortune de l'œuvre, en quelque sorte) ; 2) les lectures critiques (la réception journalistique et universitaire) ; et 3) ce qu'il est convenu d'appeler les " représentations » de l'étranger dans cette œuvre. En l'occurrence, l'image que Claude Simon a transmise dans son œuvre d'une certaine Espagne, à un moment donné de l'Histoire, et qui

\footnotetext{
${ }^{1}$ Universitat Autonoma de Barcelona.
} 
a motivé sans doute certaines prises de position par rapport à ses romans. Tout cela, bien sûr, brièvement et de façon fragmentaire.

\section{Les traductions des romans de Claude Simon en castillan et en catalan ${ }^{2}$}

Jusqu'à présent, seize romans de Claude Simon ont été traduits en castillan et quatre en catalan. C'est-à-dire à peu près tout, à part bien entendu des œuvres anciennes épuisées en français, comme $\mathrm{La}$ Corde raide ou Le Tricheur, ou bien celles qui ne se présentent pas comme des romans : La Chevelure de Bérénice, Discours de Stockholm, Femmes, Photographies, Orion aveugle, Album d'un amateur et Correspondance avec Jean Dubuffet. Pour ce qui concerne le catalan, les quatre romans traduits et publiés sont : Le Palace, Les Géorgiques, L'Acacia et Le Tramway.

\section{Les tirages}

Il est difficile de connaître les chiffres de tirage de ces différents romans. Nous avons pu trouver, çà et là, des données très partielles, qui fournissent toutefois quelques ordres de grandeur incomplets. On sait, par exemple, que Les Géorgiques, traduites en castillan en 1986 chez Seix Barrai, ont été tirées à 4000 exemplaires $^{3}$, ce qui n'est pas mal du tout, d'autant que le roman a été réédité ensuite (en 2002), ce qui laisserait entendre que ces premiers 4000 exemplaires ont été épuisés. On a pu savoir également que la première édition d'Histoire (Seix Barrai, 1971) avait été tirée à l'origine à 250 exemplaires, selon une indication de l'éditeur.

$\mathrm{Au}$ moment de l'attribution du prix Nobel en 1985, date à laquelle on a réédité La Route des Flandres, l'éditeur aurait encore eu

\footnotetext{
${ }^{2}$ Pourquoi parler du catalan ici ? Parce qu'on ne peut pas faire abstraction des publications dans cette langue dès lors que l'on sait que le monde de l'édition espagnole est dans une grande mesure situé dans la capitale catalane (aussi bien pour le castillan que pour le catalan, bien évidemment) et ce, pour l'ensemble de la péninsule ibérique et pour l'Amérique latine. Barcelone, référence essentielle de la " matière d'Espagne " de Claude Simon, est aussi la référence incontournable de l'édition de ses œuvres en castillan, comme elle l'est pour l'édition espagnole en général.

${ }^{3}$ Donnée que nous avons trouvée dans un article d'El País, lors de la publication de l'ouvrage.
} 
dans ses stocks 800 exemplaires du livre. Et on a affirmé aussi qu'à ce moment-là, tous romans confondus, il y avait plusieurs milliers de volumes imprimés du même auteur, mais que la plupart étaient sur les rayons des librairies, invendus.

On n'en sait guère plus, mais il est certain qu'après le Nobel, les tirages et les ventes ont augmenté sensiblement. Rien n'est sûr cependant car comme le rappelait Rafael Conte, à l'occasion, citant Jean Paulhan dans Les Fleurs de Tarbes: "Tout le monde sait de nos jours qu'il y a deux types de littératures : la mauvaise, qui est proprement illisible (c'est celle qui est beaucoup lue) et la bonne, que l'on ne lit pas. "

\section{Lieux d'édition}

Il est intéressant de noter que les premières traductions de romans de Claude Simon n'ont pas été faites en Espagne, mais en Argentine : pour Le Vent et L'Herbe, dès 1961, chez Fabril Editora, de Buenos Aires. C'est en Argentine également que sera traduit Le Jardin desplantes, en 1997 (aux éditions Libro Perfil, S.A.).

Le Sacre du printemps et Gulliver ont été publiés en castillan à Caracas, Venezuela cette fois, en 1969 et 1970 respectivement. Entre ces éditions américaines, la première traduction d'un roman de Claude Simon eut lieu en Espagne. Il s'agit de La Route des Flandres et elle date de 1967. Toutes les autres éditions ont été faites à Barcelone, pour les raisons que nous avons avancées.

\section{Les rééditions}

Si l'on en juge par les rééditions, les romans les plus vendus ou diffusés en Espagne (et dans les pays de langue espagnole, compte tenu du fait que l'édition barcelonaise est amplement diffusée en Amérique latine) seraient :

1. La Route des Flandres (1967, 1985, 1998)

2. Histoire (1971, 1986)

3. Les Géorgiques (1986, 1997, 2002), dont une édition intermédiaire cartonnée chez Planeta.

4. L'Herbe $(1961,1986){ }^{4}$

\footnotetext{
${ }^{4}$ L'édition de 1961 avait été faite en Argentine, comme nous l'avons dit plus haut.
} 


\section{Chronologie des traductions}

Les éditions argentines suivent de près les éditions françaises (trois ou quatre ans). Les éditions vénézuéliennes tardent beaucoup plus : quinze ans après pour Le Sacre du printemps et dix-huit ans pour Gulliver. Ces deux romans n'ont d'ailleurs jamais été publiés en Espagne.

L'ordre de publication des traductions en Espagne est, de son côté, intéressant à analyser :

1. La Route des Flandres (1960) 1967, sept ans après

2. Histoire (1967) 1971, quatre ans après

3. La Bataille de Pharsale (1969) 1971, deux ans après

4. Les Corps conducteurs (1971) 1973, deux ans après

5. Le Palace (1962) 1985, vingt-trois ans. En catalan en 1986, vingt-quatre ans après

6. Les Géorgiques (1981) 1986, cinq ans après

7. Le Tramway (2001) 2002, dès l'année suivante, aussi bien en castillan qu'en catalan.

Si l'on se souvient que le général Franco est mort en 1975, on comprend aisément pourquoi Le Palace n'a été publié qu'en 1985, c'est-à-dire près d'un quart de siècle après sa parution et uniquement après que Claude Simon eut reçu le Prix Nobel. Ce retard est dû à des problèmes de censure, directe ou induite, les éditeurs hésitant beaucoup à publier un roman dont on était à peu près sûr qu'il aurait des difficultés. Il convient en effet de signaler à ce propos que les premières éditions de La Route des Flandres et d'Histoire avaient été expurgées par le censeur lors de leur première publication. Celui-ci avait alors pratiqué soixante-dix coupes dans le premier et pas moins de vingt dans le second, dans des passages jugés ou trop érotiques ou ayant des connotations politiques.

À noter également que Seix Barrai, S.A., la maison d'édition qui a pris en charge le plus souvent les traductions de Claude Simon s'est spécialisée dans les ouvrages d'auteurs contemporains et, en particulier, ceux du Nouveau Roman. 


\section{Les traducteurs}

En général, le choix des traducteurs des œuvres de Claude Simon a toujours été excellent. On peut citer :

- José Escué Porta, agrégé de français, qui s'est spécialisé dans la traduction de romans contemporains. Il a traduit excellemment Histoire, Les Géorgiques, L'Acacia.

- Javier Albiñana (Le Tramway), qui a reçu plusieurs prix pour ses traductions.

- Esther Tusquets, directrice des Éditions Lumen (qui ont publié un certain nombre d'ouvrages de Claude Simon) est elle-même écrivain et a écrit plusieurs romans, dont l'un a reçu le Prix de la Ville de Barcelone.

- Manuel Angel Serrat Crespo, écrivain également, participant actif de toutes les activités culturelles francophones à Barcelone. C'est à lui qu'a échu la difficile tâche de traduire Le Palace.

Mais le plus remarquable, à ce sujet, est sans doute le fait que la première version en castillan de L'Herbe a été faite par Miguel Angel Asturias et sa femme Blanca de Asturias, pour la Fabril Editora de Buenos Aires. Peut-être un peu oublié maintenant, Miguel Angel Asturias (1899-1974) a été un auteur célèbre en son temps. Poète, romancier en plus de diplomate, ce Guatémaltèque a été lui-même prix Nobel de Littérature en $1967^{5}$.

Pour ce qui concerne le catalan, il faut rappeler que la version du Palace dans cette langue est signée par Jaume Fuster, écrivain et « activiste culturel» (c'est ainsi qu'on l'avait désigné), bien connu et malheureusement disparu il y a quelques années, à l'âge de 53 ans. Fuster a traduit différents auteurs surtout du français au catalan (mais parfois aussi au castillan) : Balzac, Rousseau, Boris Vian, Queneau, Albert Cohen. Pour sa traduction de Belle du Seigneur, précisément, il reçut le prix Crítica Serra d'Or, en 1989. Fuster fut aussi

\footnotetext{
${ }^{5}$ On ne sait trop comment interpréter que M. A. Asturias se soit chargé de traduire L'Herbe. Est-ce par pur intérêt pour ce roman ou bien pour d'autres raisons? Il faut se souvenir en tout cas que c'est durant son exil en Argentine, de 1954 à 1965, et avant qu'il soit nommé ambassadeur de son pays en France (où il avait auparavant fait un séjour d'études et rencontré André Breton), que M. A. Asturias et sa femme traduisent le roman de Claude Simon. Peut-être est-ce tout simplement à cause d'une situation économique difficile à ce moment-là. On ne sait. Certains chercheurs pensent d'autre part que la biographie d'Asturias contient nombre de zones d'ombre (un an avant le Nobel, en 1966, il avait reçu le prix Lénine de la paix). Il est mort à Madrid en 1974.
} 
un des fondateurs de l'Association des écrivains de langue catalane, dont il fut président de 1995 jusqu'à sa mort.

On s'attarde sur toutes ces données objectives pour insister sur le fait que l'œuvre de Claude Simon, mis à part les problèmes avec la censure pendant la période de la dictature, a été fort bien traitée par les éditeurs espagnols, qui lui ont procuré les meilleurs traducteurs. Cette affirmation est d'autre part corroborée par la circonstance suivante. Les Géorgiques, en catalan, ont été publiées dans une collection d'Edicions 62 et « La Caixa » qui porte pour titre : " Les meilleures œuvres de la littérature universelle» (vol. 35), dont le directeur est Joaquim Molas et les assesseurs sont J.M. Castellet et Pere Gimferrer. Pour qui connaît le monde de l'édition espagnole, ces noms seront familiers et prouveront, d'autre part, la haute opinion que le roman de Claude Simon a méritée auprès de ces spécialistes en littérature. La traduction, dans ce cas, des Géorgiques en catalan est due à Victor Compta ${ }^{6}$.

\section{La réception critique}

Nous n'avons pas dépouillé tout ce qui a été écrit à propos de l'œuvre de Claude Simon en Espagne. C'est une tâche longue et souvent compliquée, les documents originaux sont souvent hors de portée. Nous avons pu réunir, toutefois, les plus importants, croyonsnous. Ils vont nous permettre d'esquisser une analyse succincte de l'accueil des romans de notre auteur.

On distinguera trois domaines concrets :

a) les articles de la presse quotidienne (du moins de deux journaux parmi les plus lus : El País et La Vanguardia), et cela dans les vingt dernières années.

b) les articles de revues littéraires et/ou de la critique universitaire.

c) enfin, quelques thèses soutenues dans différentes universités espagnoles sur divers aspects de l'oeuvre qui nous intéresse.

${ }^{6}$ L'auteur de cette « réception » a eu lui-même le plaisir d'en rédiger une brève présentation. 


\section{Les articles de la presse quotidienne}

On ne s'est guère occupé dans la presse quotidienne, que l'on sache, de l'œuvre de Claude Simon hors des événements culturels importants, telle une tournée de conférences ou la parution d'une traduction. À part, bien sûr, l'événement majeur du Nobel de 1985.

Claude Simon était venu à Madrid et Barcelone, en 1984, invité par les Instituts Français de ces villes, pour y faire des conférences, alors que son nom avait été souvent cité, cette année-la, pour le prix suédois. Un titre de journal de cette époque se révèle particulièrement significatif, a posteriori. Claude Simon avait alors déclaré, en octobre 1984, à Rafael Conte : "Ya estoy quemado para el Nobel » [Maintenant, je suis fini pour le Nobel]. Comme on l'a vu par la suite, il était totalement dans l'erreur...

En 1985, la presse, bien entendu, s'est largement fait l'écho du Nobel, en précisant toujours, pour se donner bonne conscience sans doute, que " l'auteur était pratiquement inconnu dans son pays ».

Claude Simon est revenu, sur les mêmes lieux (Madrid et Barcelone) en 1986, lors des rééditions de certains de ses romans. Et de nouveau il a fait une visite à Barcelone, en 1989, pour la sortie des Géorgiques dans la collection catalane de prestige que nous avons signalée.

On fournit ces indications de voyages (il y en a eu d'autres) pour qu'on n'oublie pas de faire la part des choses. À ces dates, notre auteur, pratiquement inconnu auparavant, est devenu un protagoniste de l'actualité culturelle. Mais on sait que, hors de ces circonstances, il en va tout autrement en Espagne pour les écrivains étrangers?

\footnotetext{
${ }^{7}$ Dans le cadre des relations culturelles entre la France et l'Espagne, il faudrait consacrer tout un chapitre aux activités organisées dans et par les Instituts français. Sous le régime de Franco, c'étaient des îlots de liberté qui ont beaucoup fait pour la diffusion de la culture. Après la fin de dictature, les choses ont changé. Les conférences programmées d'écrivains ou autres ont eu et ont encore des sorts divers. Tel conférencier réunit un public nombreux, d'autres moins. Pour prendre des exemples concrets, on dira que Daniel Pennac a fait salle comble à Barcelone ; Robbe-Grillet, venu présenter La Reprise, réunit, pour sa part, un modeste auditoire, de même que Jean Échenoz. En revanche, Jean-Yves Tadié, venu parler de Marcel Proust, fit salle comble à l'IF de Barcelone. Comment interpréter ces données ? On ne sait. Il faudrait faire une étude, à cet endroit, si on s'intéresse à la fortune et à l'image de la littérature française à l'étranger, en Catalogne et en Espagne, plus précisément. Pour ce qui concerne Claude Simon, à chaque fois qu'il est venu, il a eu un public nombreux, mais il faut bien dire que nous étions encore sous l'influence médiatique du Prix Nobel.
} 
Des quinze articles sélectionnés dans cette catégorie des articles de la presse quotidienne, neuf proviennent d'El País, cinq de $L a$ Vanguardia, un d'El Periódico. En l'occurrence, ces journaux sont les plus diffusés, en Catalogne en particulier. À Madrid, il y en a d'autres (ElMundo, $A B C$...) qui se sont moins intéressés à Claude Simon et n'ont donné des informations, d'agences de presse pour la plupart (Efe), qu'en des circonstances particulières (Nobel, décès).

Deux articles marquants renvoient au premier événement rapporté : la tournée de conférences de Claude Simon à Madrid et à Barcelone. Sept sont en rapport avec l'attribution du Prix Nobel. Et trois concernent la deuxième visite aux Instituts Français.

Ces articles sont, le plus souvent, élogieux pour notre auteur. Rafael Conte, le spécialiste de Claude Simon en ce domaine, tout particulièrement. Bon connaisseur de la littérature française contemporaine, il analyse avec sympathie tout ce qui vient de France. C'est probablement le chroniqueur littéraire le plus enthousiaste et le meilleur connaisseur de l'œuvre de Claude Simon. Il est l'auteur de six des articles sélectionnés ${ }^{8}$.

Bien entendu, l'unanimité n'existe pas en la matière. Si l'on prend par exemple Baltazar Porcel, écrivain lui-même et chroniqueur à La Vanguardia depuis de nombreuses années (il a également été conseiller privé de l'ex-président de la Generalitat), voici ce qu'il écrivait au moment du Nobel

Je dois signaler, maintenant, que c'est à peine si Claude Simon m'intéresse comme romancier. J'ai essayé de lire, il y a un quart de siècle, Le Vent et La Route des Flandres. Je n'y suis pas parvenu. J'ai réattaqué, vers 1970, avec La Bataille de Pharsale: la même chose. Maintenant, je l'ai feuilleté, sans éprouver de changement notable dans mon état d'esprit. Je le regrette. On ne peut pas se convertir en conscience universelle, ni être parfait. La stupidité m'ennuie. ${ }^{9}$

\footnotetext{
${ }^{8}$ Il figure, d'ailleurs, au sommaire du $\mathrm{n}^{\circ} 2$ des Cahiers Claude Simon, publié après la mort de notre auteur, avec un bel article : «Un Nobel imperturbable ».

${ }^{9}$ Traduit par nous. Pour ce dernier substantif, Porcel utilise un mot castillan peu usité, estolidez (et non pas estupidez, qui existe également), sans doute pour être moins offensif, mais le résultat est le même. Du moins, la réversibilité de l'expression appliquée à son auteur est elle-même assurée.
} 


\section{Les articles de la critique universitaire ${ }^{10}$}

Dans cette catégorie, nous avons recensé trente-cinq documents ${ }^{11}$, sans être sûr toutefois d'avoir été exhaustif, d'autant plus que certains sont difficiles à trouver ${ }^{12}$.

Le premier de ces articles, datant de 1968, est de Domingo Pérez Minik $^{13}$. Il a été écrit huit ans après la publication du roman auquel il se réfère et un an après la parution de sa traduction espagnole. L'article suivant, d'Alicia Piquer, date de 1982, et celui de Rafael Conte, dans Insula, de 1986. À partir de là, les autres vont se succéder à un rythme plus ou moins constant jusqu'en 2006 (dans notre recensement). C'est-à-dire que les trente-cinq documents s'étalent sur une période de vingt-quatre ans. Il est donc permis de confirmer ici deux choses : l'inertie habituelle des universitaires (dans ce cas espagnols) au moment d'aborder la littérature (française) contemporaine, mais aussi une belle constance de leur part dans l'analyse des œuvres de certains romanciers.

Quatre des articles recensés ont été publiés dans le $n^{\circ} 4$ de Queste, revue de langue et littérature française des universités de Pau, Pays basque, Saragosse et Valence, à laquelle d'ailleurs il est assez difficile d'avoir accès maintenant.

Quatre autres ont été inclus dans le numéro 6 de la revue $L a$ Página, éditée à La Laguna, Tenerife (Canaries), et correspondent à des interventions présentées lors d'un colloque tenu à l'Université de cette ville, en 1991, auquel Claude Simon était présent. Deux des auteurs de ces articles ont beaucoup travaillé sur les textes de notre auteur et soutenu des thèses que nous évoquerons plus loin.

Deux autres articles ont été d'abord présentés comme communications au cours d'un Congrès international de traduction et d'interprétation, à Soria.

\footnotetext{
${ }^{10}$ Peut-être est-ce forcer quelque peu l'expression que de parler de "critique universitaire " au sens où on l'entend généralement, mais le fait est que les articles répertoriés ont pour auteurs des enseignants-chercheurs de diverses universités espagnoles.

${ }^{11}$ Dont la liste figure en annexe.

${ }^{12}$ Beaucoup ne sont pas repris dans les bibliographies générales des études sur Claude Simon et pas toujours, non plus, dans la bibliographie sur la littérature française, pourtant très complète, que réunit soigneusement tous les ans Alicia Yllera, de l'UNED (Université nationale de l'éducation à distance).

${ }^{13}$ En fait, cet auteur n'est pas à proprement parler un universitaire mais plutôt un critique ayant tenu pendant longtemps le feuilleton de littérature étrangère dans Insula, revista de letras y ciencias humanas.
} 
Il faut également noter que six auteurs ont publié vingt-cinq de ces trente-cinq écrits recensés (neuf, trois fois quatre et deux fois deux, respectivement) ce qui, d'une certaine manière, révèle la « spécialisation » de certains d'entre eux sur l'œuvre de Claude Simon. D'autre part, on apprécie une concentration indéniable de tous ces documents sur quelques aspects concrets. Les thèmes de la guerre et de la révolution l'emportent, suivis de près par celui de la peinture, suivi par la "mémoire » et la biographie (du moins le mot mémoire figure-t-il souvent dans les titres).

Tous ces textes constituent des études dans lesquelles n'entrent que rarement des jugements de valeur à propos de l'œuvre de Claude Simon. Il convient, toutefois, de signaler une note discordante (dans un document qui n'est pas inclus dans notre répertoire d'articles parce qu'il appartient à une histoire de la littérature, mais qui cependant est le fait d'un spécialiste connu), c'est celle de Carles Pujol ${ }^{14}$, professeur de littérature française, traducteur et romancier, qui écrit :

De son côté, le prix Nobel de 1985 Claude Simon (né en 1913) construit sur des fonds historiques des fantaisies très élaborées, qui appartiennent à la pure littérature expérimentale, de laboratoire : $L a$ Route des Flandres (Elcamino de Flandes, I960) [en fait le titre exact est La ruta de Flandes], La Bataille de Pharsale (La batalla de Farsalia, 1969). La rigueur formelle et conceptuelle se met ici au service d'une obscurité qui se trouve entre l'ineffable et le galimatias.

Comme on peut le constater, ce critique ne mentionne même pas Le Palace.

\section{Les thèses doctorales ${ }^{15}$}

Elles sont étalées dans le temps et leurs sujets confirment ce qui a été dit à propos des articles. Ils montrent bien d'autre part l'éventail de « lectures» que les romans de Claude Simon ont suscitées.

\footnotetext{
${ }^{14}$ Dans le volume 9 de $\mathbf{1}$ 'Histoire de la littérature universelle, chapitre " Des avant-gardes jusqu'à nos jours », p. 551.

${ }^{15}$ Il convient de signaler d'entrée que s'il existe bien un fichier central des thèses en Espagne, celui-ci est assez récent et toutes les thèses n'y sont pas répertoriées. Nous ne ferons état ici que de quatre thèses qui, croyons-nous, sont représentatives des approches de l'œuvre simonien de la part d'universitaires espagnols.
} 
1) Alicia piquer desvaux : La Retórica en Claude Simon [La rhétorique chez Claude Simon], 1979, Universitat de Barcelona. Directeur : Alain Verjat Massmann.

"La critique textuelle appliquée à Claude Simon, écrivain intégré dans les mouvements appelés du "Nouveau Roman" et du "Roman textuel" ", lit-on dans le résumé. Dans l'introduction sont signalées les influences et les caractéristiques de ces mouvements, ainsi que la place qu'ils occupent dans le panorama littéraire du siècle. La première partie est consacrée à l'examen des méthodes relatives à la critique textuelle dans son analyse du discours du roman et à la justification du choix de Gérard Genette, Philippe Hamon et Gaston Bachelard pour fonder l'étude sur Claude Simon. Ensuite sont abordées les techniques d'écriture dans la production simonienne, pour arriver à la conclusion de l'existence d'une structure appelée récit métaphorique. Puis on analyse la structure et le discours descriptif et l'on débouche sur l'élaboration de structures thématiques déterminées (le mythe d'Eros et Thanatos, le mythe de l'œuvre) qui correspondent au sens ultime de la production littéraire simonienne : à savoir une théorie implicite du phénomène littéraire, de la disparition du genre et de son remplacement par le texte.

2) Manuel Antonio tost planet : La narrativa de Claude Simon [Le discours romanesque de Claude Simon], 1984, Universidad Complutense de Madrid. Directeur : Daniel Poyán Díaz.

En quelque sorte, c'est à une recherche sur l'insertion du réel dans le discours romanesque de Claude Simon, plus précisément de ce qu'on y désigne par l'expression de "matière d'Espagne ", qu'est consacrée cette thèse. Après avoir situé l'œuvre et les lectures diverses (et souvent contradictoires) que l'on en a faites, on s'attache à analyser la fiction romanesque, les personnages, certaines des structures signifiantes simoniennes, des questions touchant à la perspective narrative et à la description. À la fin du premier volume figurent des " éléments d'iconographie pour une étude contrastive " (photographies) sur lesquels prennent appui une série de descriptions intégrées dans la matière d'Espagne.

Dans une annexe de 389 pages (dactylographiées) et sous le titre de "Documents pour une poétique de Claude Simon » sont réunis cinquante-trois documents (déclarations, articles, interviews) de 
Claude Simon (qui s'étalent de 1957 à 1982), dont la lecture est particulièrement intéressante. Bien entendu, entre ce qui a été vraiment dit par l'auteur et ce que les intervieweurs (ou journalistes) ont publié, il peut y avoir des discordances, ce qui oblige à prendre des précautions au moment de les citer $^{16}$. Il n'empêche, il $\mathrm{y}$ a là ample matière à réflexion

3) Elena SUÂREZ SÂNCHEZ : Influencias de la pintura en la obra de Claude Simon : barroco, impresionismo, Cézanne, 1990, Universidad de Sevilla.

Cette thèse analyse les influences de la peinture dans l'œuvre de Claude Simon sous un double rapport. D'une part, les références de l'auteur à des réalisations picturales et à des peintres concrets et, d'autre part, l'application de présupposés picturaux au domaine littéraire. Trois courants sont privilégiés dans cette recherche, en raison de leur rôle de générateurs du discours et de la fiction simonienne : le baroque, l'impressionnisme et celui représenté à lui seul par Paul Cézanne. Cette étude prétend établir l'importance de la présence de Poussin dans sa facette baroque, de Renoir, Monet et Boudin, ainsi que de Cézanne dans l'œuvre de Claude Simon, tout spécialement dans le traitement descriptif de la lumière, de la couleur, des ombres et des formes appliquées aux objets, à la végétation et aux corps.

La thèse contient également une interview accordée par le romancier à son auteur, le 10 juillet 1990 . Ont été aussi ajoutées des reproductions de tableaux que Claude Simon cite dans ses romans et d'autres qui, sans être décrits par l'écrivain, évoquent, selon l'opinion de l'auteure de la thèse, quelques-uns des paysages de ses romans. Dans tous les cas, des légendes (extraites des romans de Claude Simon) accompagnent ces illustrations.

\footnotetext{
${ }^{16} \mathrm{C}^{\prime}$ est un peu ce que l'auteur nous disait à propos de cette annexe $: \ll[\ldots]$ la réunion de mes interviews [...] que je n'ose pas trop regarder tant j'ai peur d'avoir dit des sottises " (Salses, le 14 août 1984). Avant, et en réponse à une demande de notre part au sujet de ses interventions dans diverses circonstances, il nous disait également : " Au sujet de ces conférences, justement, et de votre demande, il me faut vous dire que je n'ai jamais autorisé leur publication, car si certaines choses peuvent être dites oralement dans 1' "à peu près", c'est une autre affaire que de les fixer définitivement dans un texte imprimé sur lequel on ne peut pas revenir. Comme vous le savez, je ne suis pas un théoricien. J'ai du mal à formuler des concepts tant soit peu abstraits et les interviews que l'on m'arrache (il faut bien se concilier les journalistes !...) me font toujours, lorsque je les relis, un impression désagréable. » (Salses, le 18 mai 1983).
} 
4) Jésus CUENCA DE LA ROSA : Itinerario de la desilusión en la ficción novelesca. Aproximación a la obra de Claude Simon [Itinéraire de la désillusion dans la fiction romanesque. Approche de l'œuvre de Claude Simon], 1996, Universidad de Valladolid. Directeur : Francisco Javier Hernandez Rodriguez.

Lecture idéologique, cet itinéraire de la désillusion dévoile, selon son auteur :

1. La désillusion à propos de l'humanisme traditionnel, énergiquement dénoncé (Claude Simon n'accepte pas, ni ne reproduit, ni ne contribue à en stabiliser les schémas), situant l'homme d'une autre manière : entre et non pas au-dessus des choses, lit-on dans la thèse. Il s'agit de travailler à partir du " degré zéro de la pensée », état de nudité essentielle qui naît de la vieille illusion bourgeoise d'être quelqu'un, entendue comme "vanitas " baroque. Ce n'est pourtant pas, d'aucune manière, une tentative " in-humaine ». Le résultat de ce que fait la main de l'homme pourrait-il être inhumain ? affirme son auteur.

2. La désillusion de l'Histoire, si l'on entend comme " espoir» la proposition positive de construction d'un monde meilleur, où la suppression des contradictions finirait par abolir l'Histoire ellemême.

3. La désillusion des formes littéraires traditionnelles. De la même manière que le simple questionnement de la réalité ne la transforme pas, il s'oppose à elle du point de vue dialectique (destruction qui construit) : non pas en faisant de la théorie, mais en travaillant. À partir de l'expérience concrète des avatars d'une vie bien pleine, au moyen du travail "jusqu'à l'épuisement», le vide laissé par le questionnement et la destruction de la narration traditionnelle. Quand l'écrivain réalise sa tâche, sans aucun type d'expérience téléologique (vieux miroir aux alouettes de la « littérature engagée »), il se situe en pleine confrontation, pascalienne et disproportionnée, de l'номме et du VIDE. De toute manière, si «Orion n'atteindra jamais le soleil naissant " (Orion aveugle), « [c] haque fois que [l'écriture] change un tant soit peu le rapport que par son langage l'homme entretient avec le monde, [elle] contribue dans sa modeste mesure à changer celuici " (Discours de Stockholm). Finalement, l'auteur réunit une argumentation pour démontrer l'attitude positive d'Orwell, face à l'attitude négative de Claude Simon par rapport à la situation espagnole. 
Trente-cinq articles et quatre thèses qui traitent, donc, de manière complémentaire et approfondie, plusieurs des constellations thématiques de l'univers romanesque simonien : les aspects rhétoriques (ou de la poétique) de l'œuvre, les relations peinture-littérature, la thématique espagnole. Mais ces études laissent de côté d'autres aspects amplement traités ailleurs, car telle est sans doute une des caractéristiques des grandes œuvres, que de permettre des lectures diverses sur des aspects généraux, fragmentaires ou concrets.

\section{Réserves}

Un élément que l'on retrouve souvent, du moins dans les articles, est qu'ils insistent sur l'« appartenance » de l'écrivain au Nouveau Roman, quand ce n'est pas au « mouvement du Nouveau Roman ", et qu'on en fait même parfois "le père du Nouveau Roman " (Soledad Gallego-Díaz, dans El País). Curieusement, ailleurs, on a dit à peu près la même chose de Robbe-Grillet.

Or on sait que cette caractérisation, en Espagne du moins, a toujours été sentie comme plutôt péjorative, parce que le Nouveau Roman - disait-on - constitue une négation de l'humanisme, parce qu'il détruit le roman, efface le personnage, se perd dans les descriptions objectales, etc. Il n'y a pas si longtemps, Mario Vargas Llosa, dans un article où il exprimait sa nostalgie du Paris de ses rêves de jeunesse («Cuando Paris era una fiesta " [Quand Paris était une fête]) n'hésitait pas à justifier son désenchantement par «l'indigence des arts et des lettres dans la France contemporaine ", et de rappeler également que " [...] le nouveau roman de Claude Simon, Robbe-Grillet, Nathalie Sarraute et compagnie, à la mode à ce moment-là [les années soixante], allait passer comme feu follet sans laisser beaucoup de traces $»^{17}$.

Il est vrai que l'Espagne, du point de vue de l'histoire littéraire, est tard sortie d'une période de " réalisme social " engagé, pour se lancer dans un engouement sans faille pour le « réalisme magique ou fantastique » de la littérature du boum latino-américain, et que dans ces conditions, il était assez difficile que l'on y apprécie objectivement les « qualités» des romans des Éditions de Minuit ${ }^{18}$.

\footnotetext{
${ }^{17}$ El País, 19.03.2002.

${ }^{18}$ En fait, il n'y a guère que deux ou trois noms de romanciers espagnols chez lesquels on peut déceler des influences du Nouveau Roman : Juan Benet (en castillan), Mercè Rodoreda (en catalan).
} 
Mais n'en déplaise à M. Vargas Llosa, l'œuvre de Claude Simon a fait son chemin en Espagne si l'on en juge, du moins, par l'intérêt des universitaires autochtones ; qui plus est, elle est de mieux en mieux appréciée, pourrait-on dire, à mesure que l'on avance dans le temps. Une seule ombre au tableau, à notre avis : sa " thématique espagnole ", parfois mal perçue, autant par les critiques espagnols que par ceux d'autres horizons.

\section{Précisions ultimes}

Concernant cette réception de la "matière d'Espagne " de Claude Simon (et non pas de son œuvre en général), il convient d'en préciser, en conclusion, quelques aspects récurrents.

D'un côté - et pour la plupart des lecteurs non espagnols - cette matière est massivement celle de la " guerre d'Espagne ", avec toutes les connotations et tous les malentendus que cette identification globale peut comporter. Cela avait déjà été bien mis en évidence lors du Colloque de Cerisy en 1974, mais nous avons encore trouvé récemment des interprétations aberrantes, situant le cadre du Palace en Amérique latine, par exemple. La notice amplement documentée d'Alastair B. Duncan dans l'édition des Euvres de Claude Simon dans la Pléiade, a remis un certain nombre de choses au point.

De l'autre, les représentations que les romans de Claude Simon donnent de l'Espagne choquent parfois les lecteurs espagnols actuels. Ils trouvent vraiment trop « noire » cette représentation de leur pays... Ils ont du mal à comprendre comment est traitée cette «matière " par un écrivain français, renvoyant parfois à des textes d'un auteur britannique et se référant à une certaine partie de l'Espagne, à un moment donné d'une histoire ancienne de près de trois quarts de siècle, eux qui savent à peine qui était Franco, et qui ont d'autant moins d'informations sur l'époque que l'on a tout fait, en Espagne, pour oublier ces épisodes. La fortune de la formule «matière d'Espagne" (à l'image de celle de Bretagne, par la rime) ne doit pas faire oublier que, plutôt que d'Espagne, c'est de Barcelone qu'il s'agit.

Pour ce qui nous concerne, nous avons essayé à plusieurs reprises d'expliquer que lorsqu'on se réfère à cette thématique, il faut prendre garde à ne pas se fourvoyer. Si les livres de Claude Simon se déroulent bien sur fond de guerre (civile, pour la plupart d'entre 
eux ; et napoléonienne en partie pour Les Géorgiques, ce que les Catalans du Sud appellent la Guerra del Frances), il ne faut pas en conclure - ce qui a été fait trop souvent - que le contexte immédiat est celui de la Guerre d'Espagne (1936-39), et éviter les comparaisons trompeuses avec d'autres textes se rapportant eux, vraiment, à ces événements. Cette précision est d'autant plus nécessaire que des erreurs d'appréciation sur ces questions ont pu jouer un rôle en ce qui concerne certaines " lectures aberrantes ", en deçà et au-delà des Pyrénées (et dans les deux sens).

On nous permettra donc, pour finir, de faire une brève incursion dans l'étude des sources. D'un point de vue strictement littéraire, ce n'est sans doute guère intéressant. Ce l'est davantage, à notre avis, dès lors qu'on s'intéresse à la réception d'une œuvre : vérifier l'inscription de la réalité dans la fiction peut être utile.

Claude Simon n'a fait qu'un bref séjour à Barcelone, en septembre 1936. Bref, mais intense et inoubliable. Il l'a dit de manière enthousiaste, à plusieurs reprises :

Barcelone aux mains des anarchistes! Quel événement ç'a été pour moi ! Je me disais : c'est fou, c'est dingue, c'est impossible, mais que je crève dans les trois jours, cela vaut pour avoir vécu et connu ça! [...] Vous savez, toutes les " aliénations » dont parle Marx, la propriété, l'inégalité et le reste... Eh bien! elles étaient supprimées. On se disait, c'est impossible, cela ne peut pas durer, cela ne va pas durer, c'est délirant! Mais c'est sensationnel. Voir cela et crever. [...] On avait ce sentiment aussi à Barcelone ${ }^{19}$.

Il a ensuite suivi les événements espagnols avec beaucoup d'attention, du dehors. Si l'on en croit les références contenues dans $L e$ Palace, il a dû retourner à Barcelone en $1951^{20}$. Et puis, sans doute, il a dû beaucoup lire entre-temps (Homage to Catalonia, d'Eric Blair ${ }^{21}$ ) et ensuite Pierre Broué et Emile Témime (en particulier dans leur Histoire de la Guerre d'Espagne, aux Éditions de Minuit, coll. «Argu-

\footnotetext{
${ }^{19}$ Dans Madeleine Chapsal : « Entretien, Claude Simon parle », L'Express, 5 avril 1962, p. 32.

${ }^{20}$ On a dit plutôt en 1953, mais nous continuons à penser, en nous fondant sur des repères du Palace, précisément, que cela pourrait bien être en 1951.

${ }^{21}$ Claude Simon a pu consulter l'ouvrage, en son jour, et en anglais, avant la traduction française.
} 
ments »), avant de découvrir les " papiers » de son ancêtre le général d'Empire, Lacombe Saint-Michel, qui avait été gouverneur militaire de Barcelone en 1811.

Car, après la Révolution anarchiste (les mois suivant juillet 1936), ce sont les événements sanglants dels Fets de Maig (1937) qui constituent l'arrière-fond des fictions simoniennes, avec en alternance, les épisodes également policiers vécus par le gigantesque général. On se souvient que ces événements de 1937 avaient opposé anarchistes et trotskystes d'un côté et, de l'autre, les forces du gouvernement républicain de la Generalitat, sous influence communiste.

Et tout cela se passe sur les Ramblas, à Barcelone, cette ville qui, comme le soulignait peu de temps avant sa mort Manuel Vazquez Montalbán, se référant à la vocation littéraire de la cité comtale et " capitale de l'arrière-garde républicaine ", "a posé pour Orwell \{Hommage à la Catalogne), Malraux (L'Espoir), Claude Simon, qui donna un nom d'hôtel, Le Palace, au roman qui, probablement, lui fit décerner le Prix Nobel $»^{22}$.

Ailleurs, Vazquez Montalbán affirme encore que « [p]eu de villes ont réussi à poser pour un chef-d'oeuvre comme La Marge de [Pieyre de] Mandiargues ou ont vu baptiser un de ses quartiers, le chinois (el Barrio chino) par [Francis] Carco ou ont été vécues d'une manière aussi maudite, comme voleur et homosexuel, par saint [Jean] Genet, dans leJournal d'un voleur, mémoire de la Barcelone du Parallèle et de la marginalisation. »

Or de tout cela, pas plus d'ailleurs que le français, le lecteur espagnol ordinaire n'a qu'une vague conscience et n'est donc pas toujours à même de situer et contextualiser des romans, ceux qui traitent, sinon d'Espagne, du moins de la " matière catalane " et de Barcelone, plus précisément. Il y viendra sans doute, croyons-nous, dans la mesure surtout où il semble bien qu'enfin on commence maintenant à vouloir récupérer chez nous aussi la mémoire historique. 


\section{Bibliographie « espagnole » sur Claude Simon ${ }^{23}$}

\section{Articles (de chercheurs universitaires)}

SUÂREZ SANCHEZ, Elena (2006): « Nicolas Poussin en Claude Simon : Fabular sobre la pintura ", Texto y Lenguajes. Granada, Espafia. Comares. Vol. 1. Pag. 249-267.

TOST PLANET, Manuel (2006) : «Topographie partielle d'une ville "vouée à la violence" : Claude Simon et Barcelone ». La Méditerranée à feu et à sang. Poétique du récit de guerre. Textes réunis par Paul Carmignani, Jean-Yves Laurichesse et Joël Thomas. Perpignan : Presses Universitaires de Perpignan. Collection « Études ».

SUÂREZ SANCHEZ, Elena (2005): « La guerra civil española en la obra de Claude Simon ", Cauce, Revista defilología y su didáctica, $\mathrm{n}^{\circ} 28$, pp. 387-404.

TOST PLANET, Manuel A. (2004) : " "Le Récit de l'homme-fusil", du Palace: une étrange affaire de transports... », Claude Simon : allées et venues, Actes du colloque international de Perpignan (14-15 mars 2003), études réunies par Jean-Yves Laurichesse. Presses Universitaires de Perpignan, «Cahiers de l'Université de Perpignan », $n^{\circ} 34$.

LEFERE, Robin (2002) : «Claude Simon o la imposible Revolución », La Historia de Espafia en la Literatura Francesa : una fascinación, coord, por Mercè Boixareu, Robin Lefere, pp. 733-740.

TOST PLANET, Manuel (2002) : « La impalpable $y$ protectora niebla de la memoria : Claude Simon, El tranvía », Quimera, revista de literatura, ${ }^{\circ}$ 220, septiembre, pp. 71-73.

MARTÍN HERNANDEZ, Ramiro (2000) : «Que peut-on bâtir sur les sables mouvants de la mémoire ? Quelques réflexions sur Histoire de Claude Simon ", Cuadernos de filologia francesa, n 12, pp. 145-158.

TOST PLANET, Manuel Antonio (1999) : « ; Un orden en el caos de la memoria ? Claude Simon : La Ruta de Flandes », Quimera, ${ }^{\circ} 178$, marzo, pp. 74-76.

\footnotetext{
${ }^{23}$ Les références retenues ici l'ont été selon les critères suivants : auteurs espagnols ou non, ayant publié (en français, castillan ou catalan) des textes sur Claude Simon dans des publications espagnoles ou autres (universitaires, le plus souvent) et ayant un rapport évident avec l'Espagne. Toutefois, nous n'avons pas voulu inclure ici une étude : TOST PLANET, Manuel A. (1976-77) : «En torno a un tema de historia literaria francesa : ¿Quién es quién en el Nouveau Roman ? "Filologia moderna, Madrid, Consejo Superior de Investigaciones Cientificas-Universidad Complutense, Vol. n. ${ }^{\circ}$ 50-60-61, pp. 339-366). Nous y avions tenté de situer notre auteur dans la littérature française contemporaine. Mais on ne se référait pas seulement à Claude Simon, raison pour laquelle nous l'avons laissé en marge.
} 
MARTINEZ GARCÍA, Patricia (1996): «El paisaje como metáfora de la historia : Volverás a Región de Juan Benet y Las Geórgicas de Claude Simon ", Paisaje, juego y multilinguismo, $\mathrm{X}^{\mathrm{e}}$ Simposio de la Sociedad Española de Literatura General y Comparada (Santiago de Compostela, 1821 de octubre de 1994), vol. 1, 1996, pp. 393-414.

CUENCA DE LA ROSA, Jesús (1995-1996) : « Le Palace de Claude Simon : texto y contexto en la Espana negra », Cuadernos de investigación filológica, pp. 201-214.

LEFERE, Robin (1994) : «La censura franquista de las novelas de Claude Simon ", Actas del II Coloquio sobre los estudios defilologiafrancesa en la Universidad espanola, Almagro, 3-5 de mayo de 1993, coord, por Juan Bravo Castillo, pp. 409-414.

HERNANDEZ RODRIGUEZ, Francisco Javier (1994) : «Claude Simon : historia, memoria, autobiografía ", La traducción de lo inefable (Jorge Guillén, la emoción ontológica ; Claude Simón, el diálogo de la voz interior). Actas del Ier Congreso Internacional de Traducción e Interpretación de Soria, coord. Manuel Ramiro Valderrama, Antonio Bueno Garcia, pp. 235-248.

CUENCA DE LA ROSA, Jesús (1994) : "Claude Simon ou le flux de pensée écologique », La traducción de lo inefable. Actas del Ier Congreso Internacional de Traducción e Interpretación de Soria, pp. 263-272.

LEFERE, Robin (1994) : « La traducción al español de las novelas de Claude Simon », Anales de filologia francesa, n ${ }^{\circ}$, pp. 85-92.

SUÂREZ SANCHEZ, Elena (1993): "Protagonismo del color en la narrativa de Claude Simon ", Estudios de lengua y literatura francesas, $\mathrm{n}^{\circ} 7$, Univ. de Cadiz (Ejemplar dedicado a: Colores), pp. 137-148.

LEFERE, Robin (1993) : «Naturaleza y sentidos de la autobiografia : la escritura de Claude Simon ", Escritura autobiográfica. Actas del II Seminario Internacional del Instituto de Semiótica Literaria y Teatral (Madrid, UNED, 1-3 de julio 1992), pp. 267-274.

CUENCA DE LA ROSA, Jesús (1993) : «Guerra y Literatura : Proust y Claude Simon ", Thélème : revista complutense de estudios franceses, $\mathrm{n}^{\circ}$ 4, pp. 79-87.

HERNANDEZ, Francisco Javier (1991) : «Claude Simon o el rigor narrativo ", Lapágina, $\mathrm{n}^{\circ}$ 6, pp. 3-8.

CUENCA DE LA ROSA, Jesus (1991) : "Alegorías de lo corruptible y lo finito en algunos textos de Claude Simon », Lapdgina, $\mathrm{n}^{\circ}$ 6, pp. 10-20.

BARGALLÓ, Juan (1991) : "Génesis y reflejo especular », La pdgina, $\mathrm{n}^{\circ}$ 6, pp. 21-32.

SUÂREZ SANCHEZ, Elena (1991) : «Correspondencias entre Paul Cézanne y Claude Simon », Lapdgina, ${ }^{\circ}$ 6, pp. 34-50. 
SERNA RODRIGUEZ, Angela (1990) : «Pour une analyse grammatextuelle : inscription et figuration dans l'œuvre de Claude Simon ", Ensayos de literatura europea e hispanoamericana, sous la direction de Felix Menchacatorre. San Sebastian : Universidad del País Vasco, pp. 535-542.

GASTÓN, Luis (1989): «Claude Simon-Marcel Proust : deux narrations, une mémoire. Discours romanesque, mémoire effective ». Actas del Congreso Internacional de Literatura Comparada, Univ. de Granada, pp. 199-207. TOST, Manuel (1989) : Presentación de la novela de Claude Simon, Les Geòrgiques. Barcelona, Ed. 62 i La Caixa, pp. 5-9.

SERNA RODRIGUEZ, Angela (1988 [1989]) : «L'espace simonien : un jeu avec le mur du langage », Queste ${ }^{24}, 4$, 1988, pp. 133-163.

BENOIT MORINIĖRE, Claude (1988 [1989]) : « La logique de l'histoire dans L'Herbe de Claude Simon ", Queste, 4, pp. 99-106.

DIEGO MARTINEZ, Rosa de (1988 [1989]) : « Description et peinture dans l'œuvre de Claude Simon », Queste, 4, pp. 107-120.

DOMÍNGUEZ, Antonio (1988 [1989]): « Horizontalidad y verticalidad en Le Palace ", Queste, 4, pp. 121-132.

CONTE, Rafael (1986) : "Claude Simon : pintura, música, memoria », Insula, revista de letras y ciencias humanas, $\mathrm{n}^{\circ} 470-471$, enero de 1986 , pag. 8.

TOST PLANET, Manuel A. (1986) : "Claude Simon y la materia de España ", Cuadernos de Traducción e Lnterpretación, ${ }^{\circ} 7$ (Bellaterra : EUTI), pp. 187-198.

TOST PLANET, Manuel A. (1985) : «Claude Simon : pintura y literatura ", Las Nuevas Letras, $\mathrm{n}^{\circ}$ 3-4, pp. 118-124.

TOST PLANET, Manuel Antonio (1985) : «En torno al ultimo Premio Nobel de literatura : Barcelona en la obra de Claude Simon ", La Vanguardia, Sección de Cultura (22.11).

PIQUER DESVAUX, Alicia (1982) : «L'art de la description. Démystification du réalisme », Anuario de Filología, 8, pp. 329-335.

PÉREZ MINIK, Domingo (1968) : «Los caballos de Claude Simon en La Ruta de Flandes ", Insula, n 256, marzo de 1968 (Repris dans [1973] La novela extranjera en Espana, Madrid : Talleres de Ediciones Josefina Betancor, pp. 44-50.)

${ }^{24}$ Queste est une revue de langue et littérature françaises des Universités de Pau, Zaragoza, Vitoria y Valencia. Le numéro 4, de 1988, publié en 1989, réunit donc ces articles sur Claude Simon mais aussi sur d'autres écrivains. 


\section{Livre}

TOST PLANET, Manuel A. (1989) : Claude Simon. Novelas « españolas » de la guerray la revolución. Barcelona : Ediciones Peninsula, Col. Nexos (204 pages). 